\title{
Low postoperative dietary intake is associated with worse functional course in geriatric patients up to 6 months after hip fracture§
}

\author{
Sabine Goisser ${ }^{1 *}$, Eva Schrader ${ }^{1}$, Katrin Singler ${ }^{1,2}$, Thomas Bertsch ${ }^{3}$, Olaf Gefeller ${ }^{4}$, Roland Biber ${ }^{5}$, \\ Hermann-Josef Bail ${ }^{5}$, Cornel C. Sieber ${ }^{1,6}$ and Dorothee Volkert ${ }^{1}$ \\ ${ }^{1}$ Institute for Biomedicine of Aging (IBA), Friedrich-Alexander-Universität (FAU) Erlangen-Nürnberg, Kobergerstraße 60, \\ D-90408 Nuremberg, Germany \\ ${ }^{2}$ Department of Geriatrics, Klinikum Nürnberg, Paracelsus Medical University, Professor-Ernst-Nathan-Straße 1, D-90418 \\ Nuremberg, Germany \\ ${ }^{3}$ Institute of Clinical Chemistry, Laboratory Medicine and Transfusion Medicine, Central Laboratory, Klinikum Nürnberg, \\ Paracelsus Medical University, Professor-Ernst-Nathan-Straße 1, D-90418 Nuremberg, Germany \\ ${ }^{4}$ Institute for Medical Informatics, Biometrics und Epidemiology, Friedrich-Alexander-Universität (FAU) Erlangen-Nürnberg, \\ Waldstraße 6, D-91054 Erlangen, Germany \\ ${ }^{5}$ Department of Trauma and Orthopaedic Surgery, Klinikum Nürnberg, Paracelsus Medical University, Breslauer Straße \\ 201, D-90471 Nuremberg, Germany \\ ${ }^{6}$ Department of Internal Medicine and Geriatrics, Krankenhaus Barmberzige Brüder, Prüfeninger Straße 86, D-93049 \\ Regensburg, Germany
}

(Submitted 12 October 2014 - Final revision received 12 March 2015 - Accepted 27 March 2015 - First published online 20 May 2015)

\section{Abstract}

We examined the relationship between postoperative dietary intake (DI) of geriatric hip fracture (HF) patients and their functional and clinical course until 6 months after hospital discharge. In eighty-eight HF patients $\geq 75$ years, postoperative DI was estimated with plate diagrams of main meals over four postoperative days. DI was stratified as $>50,>25-50, \leq 25 \%$ of meals served. Functional status according to Barthel index (activities of daily living) and patients' mobility level before fracture, postoperatively, at discharge and 6 months later were assessed and related to DI levels. In-hospital complications were recorded according to clinical diagnosis. Associations were evaluated using $\chi^{2}$ and Kruskal-Wallis tests, and repeated-measures ANOVA and ANCOVA. Postoperatively, 28\% of participants ate $>50 \%, 43 \%$ ate $>25-50 \%$ and $28 \% \leq 25 \%$ of meals served. Irrespective of pre-fracture functional status, patients with DI $\leq 25 \%$ had significantly lower Barthel index scores at all times after surgery (all $P<0.05$ ) and ANOVA revealed a significant time $\times$ DI interaction effect $(P=0.047)$ on development of Barthel index scores that remained significant after adjustment for potential confounders. Patients with DI $>50 \%$ more often had regained their pre-fracture mobility level than those with DI $\leq 25 \%$ at discharge ( $>50 \%: 36 \%$; $>25-$ 50\%: 10\%; $\leq 25 \%$ : $0 \% ; P=0.001)$ and 6 months after discharge (88; 87; $68 \% ; P=0.087)$ and had significantly less complications (median 2 (25th-75th percentile $1-3) ; 3$ (25th-75th percentile $2-4) ; 3$ (25th-75th percentile $3-4$ ); $P=0 \cdot 012$ ). To conclude, geriatric HF patients had very low postoperative voluntary DI and thus need specific nutritional interventions to achieve adequate DI to support functional and clinical recovery.

Key words: Postoperative dietary intake: Hip fracture patients: Geriatric patients: Functional recovery: Functional course

The increasing number of hip fractures (HF) in older people is a major health care concern ${ }^{(1)}$, having drastic consequences on functional and self-care abilities of those affected $^{(2-4)}$. Although some patients are able to achieve almost complete functional restoration, the majority suffers from permanent disability and decline of autonomy and quality of life ${ }^{(2-4)}$. Considering that the average HF patient is increasingly dependent and in need of care even before $\mathrm{HF}^{(5)}$, huge hospital and

Abbreviations: ADL, activities of daily living; DI, dietary intake; HF, hip fracture; T1, before fracture; T2, postoperatively; T3, at hospital discharge; T4, 6 months after hospital discharge.

*Corresponding author: S. Goisser, fax +49 911530296 151, email sabine.goisser@fau.de

\Conference presentations: Preliminary results of this study have been presented as poster at the 36th ESPEN Congress on Clinical Nutrition and Metabolism 2014 in Geneva, Switzerland and at the 10th International Congress of the European Union Geriatric Medicine Society 2014 in Rotterdam, the Netherlands. 
long-term costs of care are to be expected ${ }^{(1)}$. Successful interventions to support the restoration of functional capacity after $\mathrm{HF}$ are required, and they should address nutrition as one of the important modifiable factors influencing the regeneration process $^{(2,6,7)}$.

Malnutrition is highly prevalent in the older hospitalized HF population $^{(8-11)}$, has amplifying effects on age-related decline in muscle mass and strength (sarcopenia) ${ }^{(6,11,12)}$ and is associated with an increased risk of complications, rehospitalisation and mortality in these patients ${ }^{(6,9-11,13)}$

Thus, we previously examined functional and clinical trajectories according to pre-fracture nutritional status in a sample of geriatric HF patients comprising all cognitive and functional levels $^{(14)}$. In these patients, a worse pre-fracture nutritional status was constantly associated with a worse functional status from before fracture to 6 months after hospital discharge, but unexpectedly not with significantly worse trajectory of functional recovery or worse clinical course.

It has been repeatedly shown that the nutritional status of older patients often deteriorates during hospitalization for various reasons $^{(11,15-20)}$, with insufficient dietary intake (DI) being one of the main contributing factors ${ }^{(11,21-23)}$.

Low DI has been reported in geriatric HF patients postoperatively $^{(24-29)}$, throughout hospital stay $^{(8,19,20,30-32)}$ and during rehabilitation ${ }^{(18,33)}$. In these patients, low voluntary DI has been linked with deterioration of nutritional status $^{(19,20,25)}$ and worse clinical outcome $e^{(24,27-30,33)}$ compared with patients receiving interventions to increase DI. Li et al. ${ }^{(9)}$ reported worse functional trajectories over 12 months after hospitalisation in geriatric HF patients being associated with worse nutritional status as assessed at the end of hospital stay.

Most studies assessing the association of DI with functional development after HF could not show an effect of higher DI on functional recovery in older HF patients ${ }^{(26,28,30,33)}$, whereas some did find such effects ${ }^{(18,32)}$. However, these studies were constricted to hospital stay ${ }^{(26,28,30,32)}$ or started only in rehabilitation after acute hospital stay ${ }^{(18,33)}$. Some of them also excluded relevant patient subgroups like functionally or cognitively impaired patients ${ }^{(18,32,33)}$ or malnourished persons ${ }^{(30,33)}$, although these patients constitute a considerable portion of those sustaining $\operatorname{HF}^{(8,10,26,32,34,35)}$. To the best of our knowledge, there is no previous study examining the association of voluntary postoperative DI with functional and clinical course of older HF patients over a period of 6 months after hospitalisation and including all relevant patient subgroups.

We therefore assessed this relationship in an HF patient sample including all cognitive and functional levels under the hypothesis that low postoperative DI would be associated with worse postoperative functional and clinical course.

\section{Methods \\ Study design and recruitment}

For this observational study with follow-up after 6 months, all patients aged $\geq 75$ years with surgically repaired proximal femoral (subtrochanteric, pertrochanteric and femoral neck) fracture (in this text further referred to as hip fracture (HF)) consecutively admitted to the Department of Trauma and Orthopaedic Surgery of a large urban, maximum care hospital (Klinikum Nürnberg, Germany) from October 2011 to June 2012 were asked to participate. Exclusion criteria were a presumed terminal state, transfer from other hospitals or hospital departments, German-language skills insufficient for answering questions, known cancer-related pathological fractures, cancer with acute radiation or chemotherapy, postoperative transfer to another department or intermediate care unit for more than $72 \mathrm{~h}$ (i.e. unavailable for assessment). For this analysis, only patients with complete follow-up assessment and DI data were included.

This study was conducted according to the guidelines laid down in the Declaration of Helsinki and all procedures involving patients were approved by the ethics committee of the Friedrich-Alexander-Universität Erlangen-Nürnberg, Germany. Written informed consent was obtained from every participant or participants' legal custodian. Standardized data acquisition was carried out by three trained persons at four points of time: addressing the situation before fracture (T1, as retrospective interview), within $48 \mathrm{~h}$ postoperatively (T2), at discharge from hospital (T3) and in a follow-up telephone interview 6 months after discharge (T4). In addition, patients' medical charts were reviewed and proxies or caregivers were contacted for additional information when necessary, for example, in patients with dementia or delirium.

\section{Baseline characteristics}

Participants' demographic characteristics (age, sex, housing situation) were obtained from patients or their proxies in personal interviews, and clinical characteristics (number and type of co-morbidities and medication) from medical documentation. Co-morbidities were grouped according to the German version of the ICD-10 system (International Classification of Diseases, 10th revision) ${ }^{(36)}$. The severity of co-morbid conditions was determined with the Charlson Comorbidity Index (0-37 points $)^{(37)}$. Polypharmacy was defined as taking more than five different drugs per day.

Nutritional status before HF (T1) was determined retrospectively at T2 by interviewing participants or proxies using the Mini Nutritional Assessment-Long Form (0-30 points ${ }^{(8,38)}$. Depending on total Mini Nutritional Assessment score, patients were classified as well nourished ( $>23.5$ points), at risk of malnutrition $(17-23.5$ points) or malnourished $(<17$ points). Information on previous weight loss and previous reduction of DI was taken from Mini Nutritional Assessment questions $\mathrm{A}$ and $\mathrm{B}$, respectively, and dichotomized as weight loss $\geq 1 \mathrm{~kg}$ in the previous 3 months (yes/no) and any reduction of DI in the previous 3 months (yes/no).

Participants' postoperative cognitive status was evaluated by Mini Mental State Examination (0-30 points) ${ }^{(39)}$ at T2. Patients were considered as having no cognitive impairment if achieving $>26$ points, mildly to moderately impaired with 10-26 points and severely impaired with $<10$ points $^{(40)}$ or if the test could not be completed for cognitive reasons. Depressive symptoms at T2 were assessed with the fifteen-item Geriatric 
Depression Scale $(0-15 \text { points })^{(41)}$, with a result $\geq 6$ points indicating presence of symptoms.

\section{Dietary intake}

Postoperative DI was determined with the plate diagram method $^{(42,43)}$. Starting with breakfast on the first day after surgery, each participants' DI was estimated over $4 \mathrm{~d}$ by trained study staff after each main meal and recorded as having eaten all (100\%), three-quarters (75\%), half (50\%), onequarter $(25 \%)$ or nothing $(0 \%)$ of the whole meal served (Fig. 1), irrespective of which single components had been eaten or left over. Snacks and oral nutritional supplements were not considered. For all patients with at least eleven of twelve possible plate diagrams completed, the overall DI was calculated as the estimated intake percentage of all meals recorded and then stratified as DI $>50 \%,>25-50 \%$ or $\leq 25 \%$.

\section{Functional status and functional course}

The ability to perform basic activities of daily living (ADL) was assessed for T1, T2, T3 and T4 with the ten-item Barthel index score $(0-100 \text { points })^{(44)}$. Postoperative ADL loss in Barthel index points (T2 - T1), residual ADL loss at T3 (T3 - T1) and total residual ADL loss at T4 (T4 - T1) were calculated. Participants' mobility levels (bedridden or chair-bound, mobile only with helper (with or without assistive devices for locomotion) or mobile alone (with or without assistive devices for locomotion)) at T1, T2, T3 and T4 were asked and recorded.

\section{Clinical course}

In-hospital mortality, number and type of complications during hospital stay (including all clinically diagnosed conditions occurring in hospital that needed treatment or affected the patients' management) and length of stay were extracted from medical documentation. At follow-up (T4), information about mortality and rehospitalisation of participants was gathered from patients or proxies.

\section{Data analysis and statistics}

Continuous data were examined for normal distribution by exact Kolmogorov-Smirnov test and are presented as

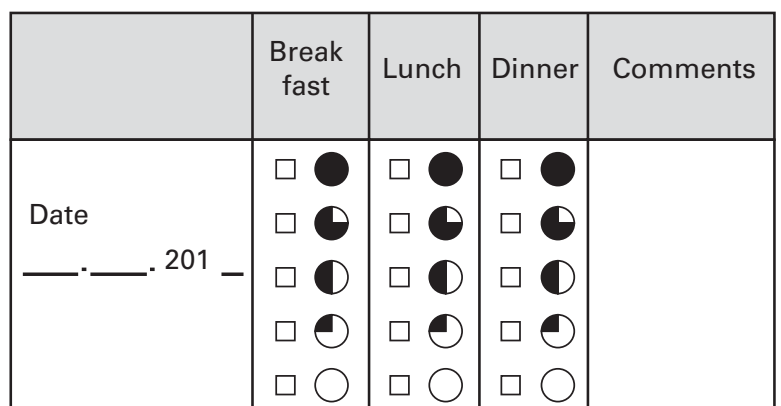

Fig. 1. Plate diagram record sheet for $1 \mathrm{~d}$. •, All; $\bullet, 3 / 4 ; 0,1 / 2 ; 0,1 / 4$; $O$, nothing eaten. median and interquartile range (25th-75th percentiles) since most parameters were distributed non-normally. Categorical data are presented as absolute numbers and percentages. Data were stratified into three groups according to DI level ( $>50 \%,>25-50 \%$ or $\leq 25 \%$ ). Differences in continuous variables between the groups were tested for significance with Kruskal-Wallis test, followed by pairwise exact MannWhitney $U$ test. Prevalence rates of categorical variables were compared between DI groups and tested for statistically significant differences with $\chi^{2}$ or modified Fisher's exact test, as appropriate. A $P$-value $<0.05$ (two-sided) was considered statistically significant after correction for multiple comparisons with the Benjamini-Hochberg procedure ${ }^{(45)}$.

ANOVA with repeated measures was performed to assess whether there was a significant difference between DI groups in change of ADL scores over time. Polynomial contrasts were used to account for the unequal time intervals between measurements. When a significant Mauchly's test indicated that the assumption of sphericity had been violated, df were corrected using Huyhn-Feldt estimates of sphericity. The explained variance (\%) is based on partial $\eta^{2}$. For each significant effect, post hoc analysis between DI groups was performed using the simple Bonferroni correction to control for the effect of multiple comparisons.

In addition, the ANOVA model was extended to an ANCOVA with repeated measures to adjust for the influence of potentially confounding factors. The confounders taken into consideration were included in the model as covariates if continuous (age, number of co-morbidities, Mini Nutritional Assessment points), otherwise as between-subjects factor (sex, postoperative cognitive impairment according to Mini Mental State Examination score (dichotomized to: no or mild cognitive impairment (20-30 points)/moderate to severe cognitive impairment $(<20$ points $))$ ). All potential confounders showing a significant interaction effect with ADL levels over time and an impact $>10 \%$ on the time $\times$ DI group interaction effect were included in the final model.

All statistical analyses were performed using IBM SPSS Statistics for Windows, version 21.0.0.1 (IBM Corporation).

\section{Results}

\section{Study sample}

During the 9-month recruitment period, 236 persons with proximal femoral fractures were admitted to the Department of Trauma and Orthopaedic Surgery; of which, 132 were eligible. Of the 132 persons, 117 (89\% of eligible patients) agreed to participate (Fig. 2). At follow-up (T4), seventeen persons had died (mortality rate 14.5\%) and three refused further participation, resulting in ninety-seven patients $(83 \%$ of study participants) with complete follow-up assessment. After excluding nine participants with incomplete DI data (for various reasons, e.g. plate cleared up before DI estimation or meal shared with relatives), eighty-eight patients ( $75 \%$ of initial study participants) remained for the present analysis. No significant differences regarding age, sex, type of fracture or other baseline characteristics were found between the 


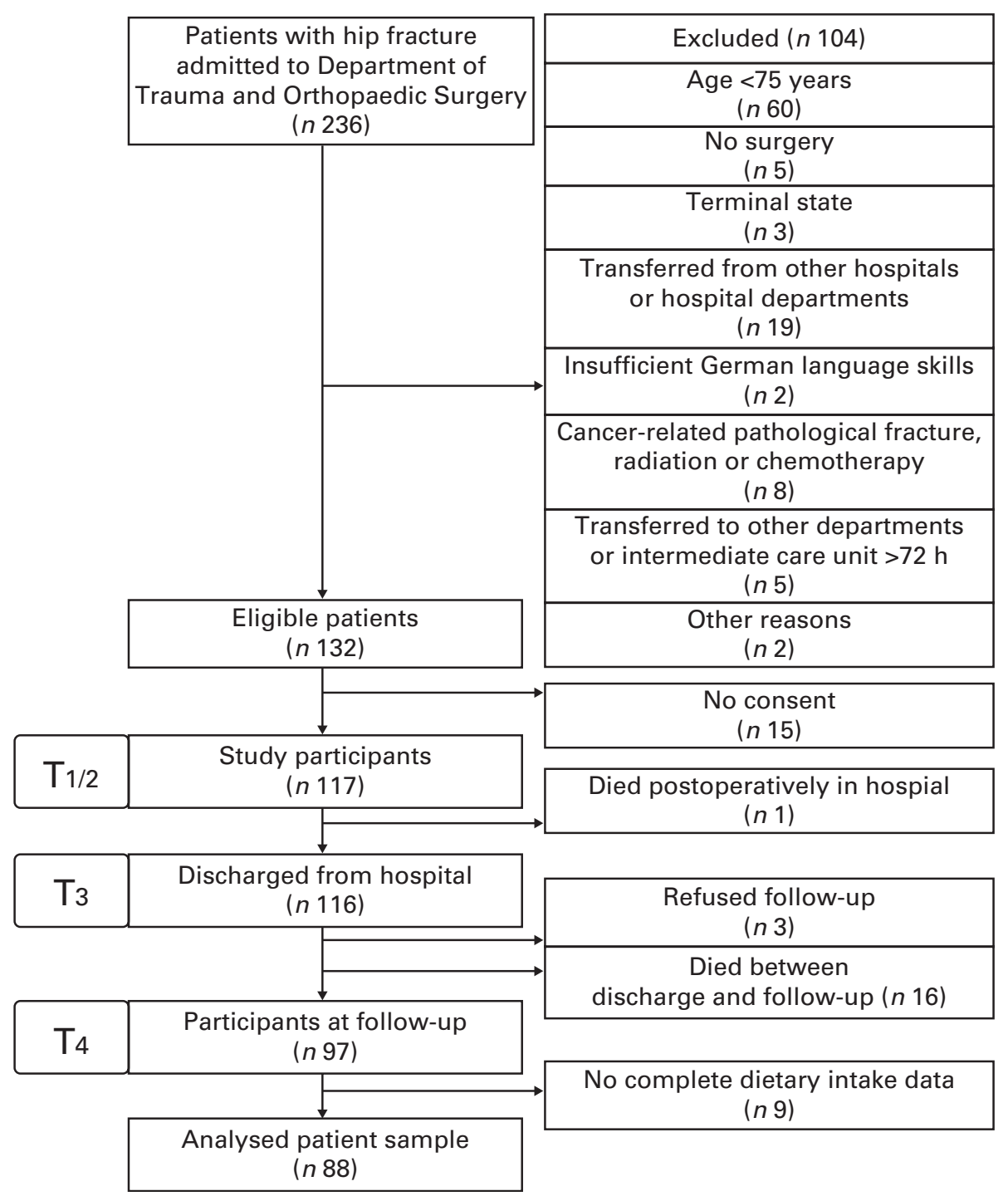

Fig. 2. Flow chart of study participation. T1, before fracture; T2, postoperatively; T3, at hospital discharge; T4, 6 months after T3.

final study sample ( $n$ 88) and those who refused participation at baseline ( $n$ 15). Those lost for analysis ( $n$ 29) were also not significantly different in baseline characteristics to the final study sample, with the exception that these persons more often were male (lost for analysis $38 \% v$. study sample $18 \%$; $P=0.042$ ), more often had malignant tumours (14 v. $0 \%$; $P=0.003)$ and had a higher Charlson Comorbidity Index score (median 3 (25th-75th percentile 2-4) v. 2 (25th-75th percentile $1-3)$ points; $P=0.003)$. Mortality until T4 did not differ between the DI levels ( $n 13$ of those that had died had complete DI data; DI > 50\%: $14 \%(n 4)$; DI $>25-50 \%$ : $10 \%$ ( $n$ 4); DI $\leq 25 \%: 17 \%(n$ 5); $P=0.654)$.

\section{Baseline characteristics and dietary intake}

Eighty-one per cent of the study participants were female, mean age was 84 (SD 5) years (maximum 97 years). Before fracture, $67 \%$ were living in a private household, 9\% in assisted housing and $24 \%$ were admitted from a nursing home. All but one participant had multiple co-morbidities (median 8 (25th-75th percentile 5-11) medical conditions in addition to HF), the main co-morbidities being cardiovascular (86\%), urogenital (80\%), metabolic (67\%) and musculoskeletal diseases (55\%). At T1, 35\% of the patients were at risk of malnutrition and 17\% malnourished according to the Mini Nutritional Assessment score.

Postoperatively, $22 \%$ of the participants had no cognitive impairment, $51 \%$ mild to moderate and $18 \%$ severe cognitive impairment. Six per cent refused to be tested and 3\% were too deaf. Depressive symptoms were detected in $15 \%$, in $18 \%$ severe cognitive impairment impeded testing and again $6 \%$ refused to be tested.

Median DI was $43 \%$ (25th-75th percentile 25-52) of the meals offered (range 6-79\%). Twenty-five (28\%) participants ate on average $>50 \%$, thirty-eight $(43 \%)$ ate $>25-50 \%$ and twenty-five $(28 \%)$ patients ate $\leq 25 \%$. Only one person ate $>75 \%$. In Table 1 , the subjects' baseline characteristics are presented according to their postoperative DI level. 
Table 1. Baseline characteristics of geriatric hip fracture patients according to postoperative dietary intake (DI) (Number of patients and percentages; median values with their 25th-75th percentiles)

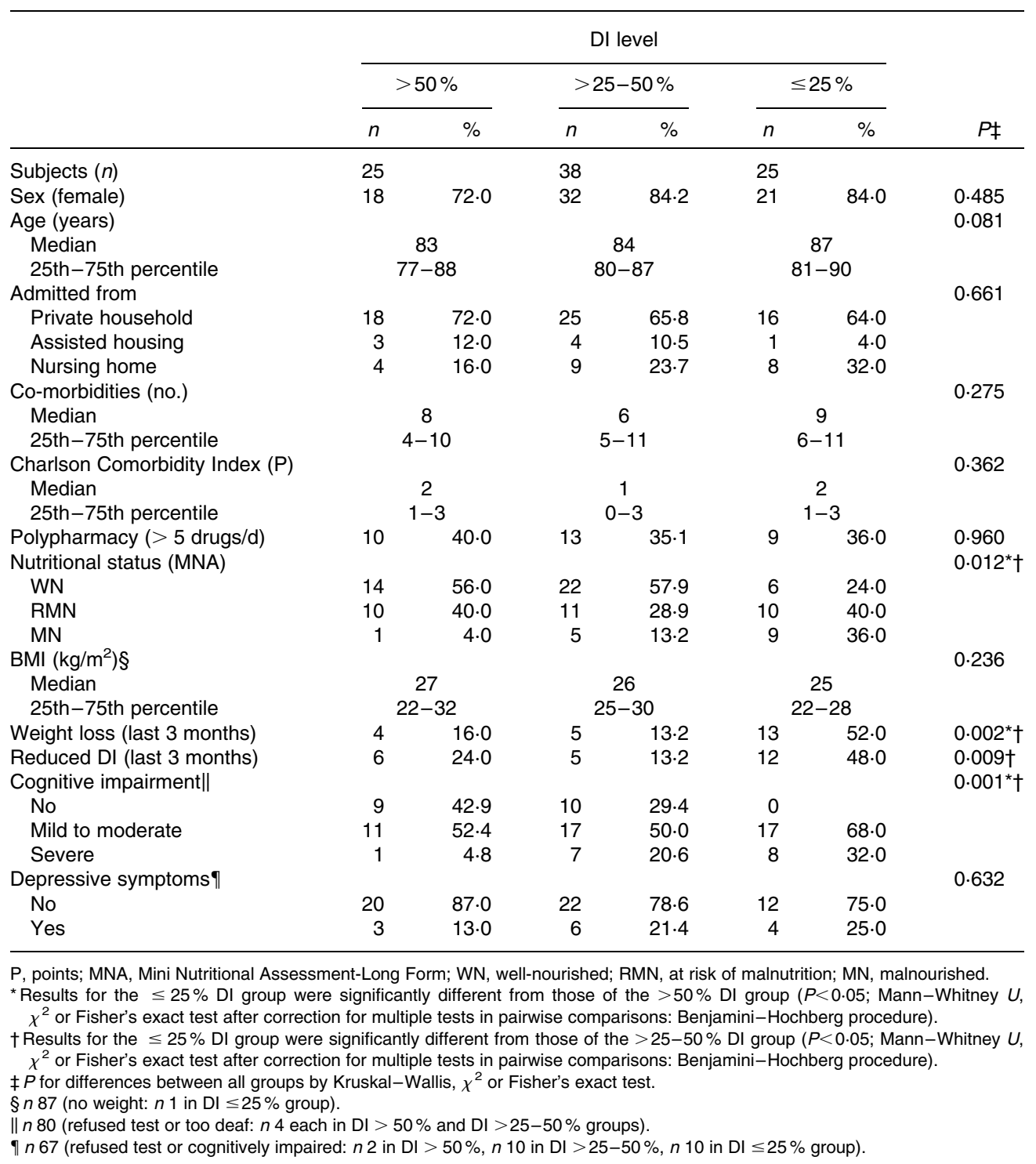

The groups were comparable regarding sex, BMI, pre-fracture housing situation, number and prevalence of co-morbidities, Charlson Comorbidity Index score and prevalence of polypharmacy at T1, but patients with DI $\leq 25 \%$ were slightly (though not significantly) older. These patients also had a significantly worse pre-fracture nutritional status according to the Mini Nutritional Assessment score and more often reported loss of weight and/or reduced DI in the 3 months before fracture than patients with higher DI. Participants with lower DI also suffered more often from cognitive impairments, and accordingly more of these patients were unable to perform the Geriatric Depression Scale (Table 1).

\section{Functional status and functional course}

Median Barthel index score for all participants was 90 (25th-75th percentile $75-95$ ) points at T1, decreased to 25 (25th-75th percentile $15-39$ ) points at T2 and then gradually increased to 40 (25th-75th percentile 25-64) points at T3 and to 75 (25th-75th percentile $30-90)$ points at T4 (Fig. 3), when $68 \%$ of patients had not regained their pre-fracture level of independence in ADL ( $\mathrm{T} 4-\mathrm{T} 1<0$ points). Thirty-four per cent had a total residual loss $>20$ points.

There was no significant difference in ADL scores between DI groups at T1 (Fig. 3). However, patients with postoperative DI $\leq 25 \%$ had significantly higher postoperative ADL loss, residual ADL loss at $\mathrm{T} 3$ and remaining total residual $\mathrm{ADL}$ loss at T4 compared with patients with higher DI (Table 2). Accordingly, patients with lowest DI had significantly lower ADL scores at all times after surgery (Fig. 3) and significantly more of these patients suffered from remaining ADL losses $>20$ points at $\mathrm{T} 3$ and $\mathrm{T} 4$ (Table 2 ).

ANOVA with repeated measures resulted in a significant time $\times$ DI group interaction $(F(5 \cdot 2)=2 \cdot 26, \quad P=0.047$, explained variance (partial $\left.\eta^{2}\right)=5 \cdot 1 \%$ ), indicating a dependence of ADL development over time from DI level. 


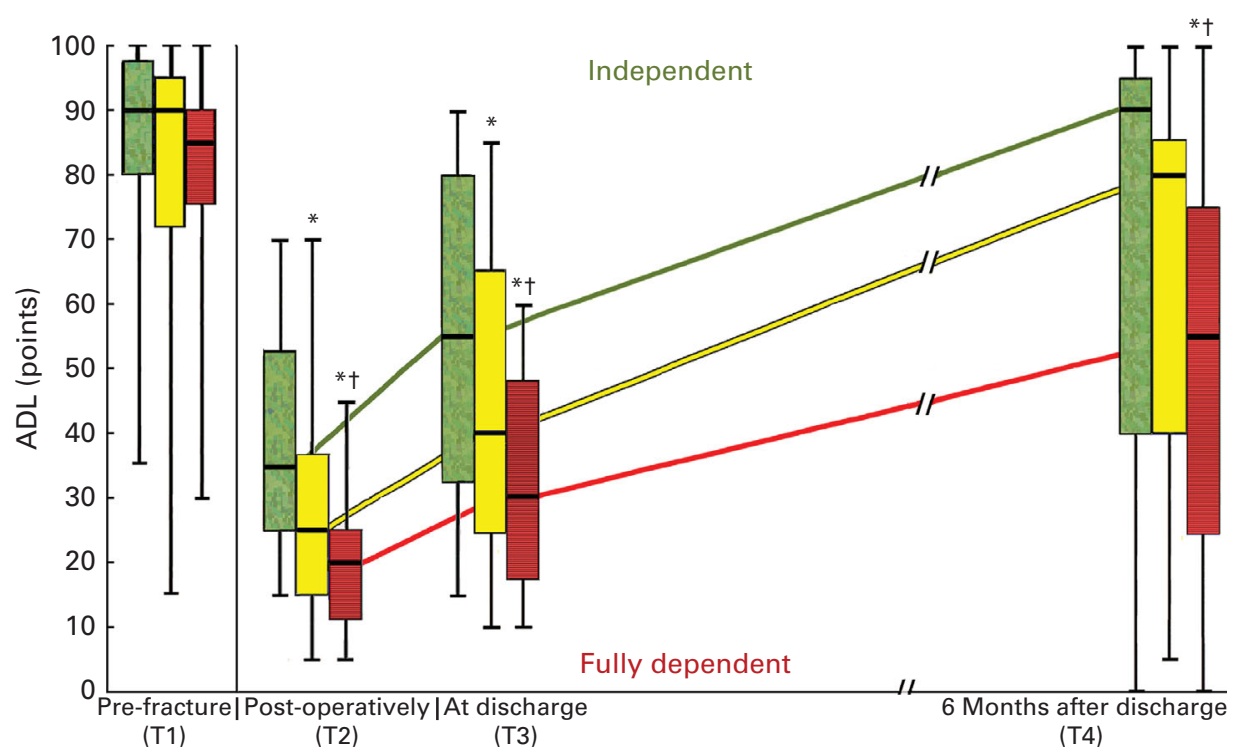

Fig. 3. Functional course of geriatric hip fracture patients as boxplots of Barthel index scores (basic activities of daily living; ADL) at four points in time according to postoperative dietary intake (DI). Data are medians, with interquartile ranges represented by vertical bars. * Median value was significantly different from that of the $>50 \%$ DI group $(P<0.05$; Mann-Whitney $U$ test after correction for multiple tests in pairwise comparisons: Benjamini-Hochberg procedure). + Median value was significantly different from that of the $>25-50 \%$ DI group $(P<0.05$; Mann-Whitney $U$ test after correction for multiple tests in pairwise comparisons: Benjamini-Hochberg procedure). $\square,>50 \%$ ( $n$ 25); $\square,>25-50 \%$ ( $n$ 38); $\square, \leq 25 \%$ ( $n$ 25). A colour version of this figure can be found online at http://www. journals.cambridge.org/bjn

Post hoc analysis of pairwise group comparisons revealed that the difference was significant only between the groups with the lowest and the highest DI $(P=0.004)$.

Of the potential confounders, number of co-morbidities $\left(F(2 \cdot 6)=4.44, \quad P=0.007, \quad\right.$ partial $\left.\eta^{2}=4.9 \%\right)$, points in Mini Nutritional Assessment $(F(2 \cdot 6)=2 \cdot 57, P=0 \cdot 020$, partial $\left.\eta^{2}=4.0 \%\right)$ and postoperative cognitive impairment according to the Mini Mental State Examination score
$\left(F(2 \cdot 6)=5.31, P=0.002\right.$, partial $\left.\eta^{2}=5.8 \%\right)$ also showed a significant interaction effect with ADL level over time. Of these, only the inclusion of Mini Nutritional Assessment points had an impact $>10 \%$ on the time $\times$ DI group interaction, increasing the effect of DI level on temporal ADL course (DI group $\times$ time: $F(5 \cdot 2)=2 \cdot 73, \quad P=0.019$, partial $\eta^{2}=6 \cdot 1 \%$; Mini Nutritional Assessment points $\times$ time: $F(2 \cdot 6)=4 \cdot 47, P=0 \cdot 007$, partial $\left.\eta^{2}=5 \cdot 1 \%\right)$.

Table 2. Changes in Barthel index scores (basic activities of daily living; ADL) of geriatric hip fracture patients from before fracture to 6 months after hospital discharge according to postoperative dietary intake (DI)

(Number of patients and percentages; median values with their 25 th -75 th percentiles)

\begin{tabular}{|c|c|c|c|c|c|c|c|}
\hline & \multicolumn{7}{|c|}{ DI level } \\
\hline & \multicolumn{2}{|c|}{$>50 \%$} & \multicolumn{2}{|c|}{$>25-50 \%$} & \multicolumn{2}{|c|}{$\leq 25 \%$} & \multirow[b]{2}{*}{$P \neq$} \\
\hline & $n$ & $\%$ & $n$ & $\%$ & $n$ & $\%$ & \\
\hline Subjects $(n)$ & \multicolumn{2}{|c|}{25} & \multicolumn{2}{|c|}{38} & \multicolumn{2}{|c|}{25} & \\
\hline Postoperative ADL loss (T2-T1) (P) & \multirow{2}{*}{\multicolumn{2}{|c|}{-45}} & & & & & $0.005^{*} \dagger$ \\
\hline Median & & & \multicolumn{2}{|c|}{-50} & \multicolumn{2}{|c|}{-65} & \\
\hline 25th-75th percentile & \multicolumn{2}{|c|}{-30 to -60} & \multicolumn{2}{|c|}{-35 to -65} & \multicolumn{2}{|c|}{-55 to -70} & \\
\hline Residual ADL loss at T3 (T3 - T1) (P) & \multirow{2}{*}{\multicolumn{2}{|c|}{-25}} & & & & & $0.008^{*}$ \\
\hline Median & & & \multicolumn{2}{|c|}{-35} & \multicolumn{2}{|c|}{-45} & \\
\hline 25th-75th percentile & \multicolumn{2}{|c|}{-15 to -40} & \multicolumn{2}{|c|}{-20 to -51} & \multicolumn{2}{|c|}{-35 to -65} & \\
\hline Patients with any residual ADL loss & 24 & $96 \cdot 0$ & 36 & 94.7 & 25 & $100 \cdot 0$ & 0.784 \\
\hline Patients with residual ADL loss $>20 \mathrm{P}$ & 13 & $52 \cdot 0$ & 26 & $68 \cdot 4$ & 22 & $88 \cdot 0$ & $0.023^{*}$ \\
\hline Total residual ADL loss at T4 (T4 - T1) $(\mathrm{P})$ & \multirow{3}{*}{\multicolumn{2}{|c|}{$\begin{array}{c}0 \\
0 \text { to }-33\end{array}$}} & & & & & $0.009^{*} \dagger$ \\
\hline Median & & & \multirow{2}{*}{\multicolumn{2}{|c|}{$\begin{array}{c}-10 \\
0 \text { to }-16\end{array}$}} & \multirow{2}{*}{\multicolumn{2}{|c|}{$\begin{array}{c}-20 \\
-5 \text { to }-53\end{array}$}} & \\
\hline 25th-75th percentile & & & & & & & \\
\hline Patients with any total residual ADL loss & 11 & $44 \cdot 0$ & 27 & $71 \cdot 1$ & 22 & $88 \cdot 0$ & $0.004^{\star}$ \\
\hline Patients with total residual ADL loss $>20 \mathrm{P}$ & 6 & $24 \cdot 0$ & 9 & $23 \cdot 7$ & 15 & $60 \cdot 0$ & $0.006^{\star} \dagger$ \\
\hline
\end{tabular}

T2, postoperatively; T1, before fracture; $P$, points; T3, at hospital discharge; T4, 6 months after T3.

${ }^{*}$ Results for the $\leq 25 \%$ DI group were significantly different from those of the $>50 \%$ DI group $\left(P<0.05\right.$; Mann-Whitney $U, \chi^{2}$ or Fisher's exact test after correction for multiple tests in pairwise comparisons: Benjamini-Hochberg procedure).

† Results for the $\leq 25 \%$ DI group were significantly different from those of the $>25-50 \%$ DI group $\left(P<0.05\right.$; Mann-Whitney $U, \chi^{2}$ or Fisher's exact test after correction for multiple tests in pairwise comparisons: Benjamini-Hochberg procedure).

$\ddagger P$ for differences among all three groups by Kruskal-Wallis, $\chi^{2}$ or Fisher's exact test. 
Regarding mobility, at $\mathrm{T} 1$, all but one participant were mobile on their own; one patient needed a helper for locomotion (Table 3). At T2, all patients were bedridden or chair-bound and had to try to start walking again with helpers and assistive devices. At T3, $85 \%$ of patients had not regained their pre-fracture mobility level: $21 \%$ were bedridden or chairbound, $66 \%$ were mobile only with helpers and 14\% were mobile on their own with assistive devices, with significantly worse mobility in patients with lower postoperative DI (Table 3), because more of them had not regained their prefracture mobility level at T3 (DI > 50\%: 64\%; DI > 25-50\%: $90 \%$; DI $\leq 25 \%$ : $100 \% ; P=0 \cdot 001)$. At T4, $2 \%$ of all patients were still bedridden or chair-bound and 16\% were mobile only with a helper. Seventeen per cent had not regained their pre-fracture mobility level, and this was still seen more often in patients with lowest postoperative DI (DI $>50 \%$ : $12 \%$; DI $>25-50 \%: 11 \%$; DI $\leq 25 \%$ : $32 \%$; $P=0.087)$.

\section{Clinical course}

Mean length of hospital stay was 13 (SD 5) d (median 11 (25th-75th percentile 9; 15) d, range 5-42d), with no difference between DI groups. Ninety-three per cent of patients suffered from at least one postoperative complication, the main problems being electrolyte imbalance (67\%), infections (49\%) and delirium (44\%). Patients with lowest DI suffered from a higher number of complications and more often had (urinary tract) infections and dehydration (Table 4). During follow-up, $46 \%$ had been readmitted to a hospital at least once, with no difference between DI groups (Table 4).

\section{Discussion}

In this observational study with 6 months follow-up, the majority of geriatric $\mathrm{HF}$ patients had an alarmingly low voluntary postoperative DI, with $72 \%$ eating only $<50 \%$ of meals served over the first four postoperative days. Patients with lowest DI had significantly lower ADL scores at all times after surgery than patients with higher DI and more of them suffered from persistent mobility losses up to 6 months after HF, irrespective of functional status before HF.

Indeed, the majority of our participants had a relatively high pre-fracture functional level compared to other studies in geriatric HF patients that also did not exclude functionally and/or cognitively impaired persons or nursing home residents ${ }^{(5,10,35,46)}$. Otherwise, the present study sample was comparable to these studies regarding distribution of $\operatorname{sex}^{(5,10,20,34,35,46)}$, fracture type ${ }^{(5,10,35,46)}, \mathrm{BMI}^{(10)}$, number and severity of co-morbidities ${ }^{(10)}$, prevalence of malnutrition and risk of malnutrition ${ }^{(10)}$, proportion of cognitively impaired patients $^{(20,34)}$ and nursing home residents ${ }^{(10,20,35)}$, but were slightly older ${ }^{(10,20,34,46)}$.

In the present study, DI was determined with plate diagrams. With this method, a patient's intake is estimated visually as a portion of the whole meal offered irrespective of exactly how much of each single component has actually been eaten. This method yields only semi-quantitative results; however, in a validation study that we have previously published as an abstract ${ }^{(43)}$, it showed high agreement with the gold standard weighing record when applied for $4 \mathrm{~d}$ in a row, and was able to correctly identify patients with low intake of energy and/or protein. The plate diagrams were filled out by specially trained and experienced study personnel with a direct view of the plate, as opposed to assessment by staff during daily routine, and thus can be deemed to be very reliable. Literature suggests that regular ward staff, which has to cope with many other tasks at the same time, has a tendency to overestimate DI of older patients ${ }^{(47)}$.

A limitation of the present study is that for this analysis only main meals could be taken into account, leading to a potential

Table 3. Mobility level of geriatric hip fracture patients before fracture, at hospital discharge and 6 months later, according to postoperative dietary intake (DI)

(Number of patients and percentages)

\begin{tabular}{|c|c|c|c|c|c|c|c|}
\hline & \multicolumn{7}{|c|}{ DI level } \\
\hline & \multicolumn{2}{|c|}{$>50 \%$} & \multicolumn{2}{|c|}{$>25-50 \%$} & \multicolumn{2}{|c|}{$\leq 25 \%$} & \multirow[b]{2}{*}{$P \dagger$} \\
\hline & $n$ & $\%$ & $n$ & $\%$ & $n$ & $\%$ & \\
\hline Subjects $(n)$ & \multicolumn{2}{|c|}{25} & \multicolumn{2}{|c|}{38} & \multicolumn{2}{|c|}{25} & \\
\hline T1 & & & & & & & 0.999 \\
\hline Bedridden or chairbound & 0 & & 0 & & 0 & & \\
\hline Mobile only with helper & 0 & & 1 & $2 \cdot 6$ & 0 & & \\
\hline Mobile alone & 25 & $100 \cdot 0$ & 37 & $97 \cdot 4$ & 25 & $100 \cdot 0$ & \\
\hline T3 & & & & & & & $0.001^{\star}$ \\
\hline Bedridden or chairbound & 2 & $8 \cdot 0$ & 7 & $18 \cdot 4$ & 9 & $36 \cdot 0$ & \\
\hline Mobile only with helper & $1 \overline{4}$ & $56 \cdot 0$ & 28 & 73.7 & 16 & 64.0 & \\
\hline Mobile alone & 9 & $36 \cdot 0$ & 3 & 7.9 & 0 & & \\
\hline T4 & & & & & & & 0.165 \\
\hline Bedridden or chairbound & 0 & & 0 & & 2 & 8.0 & \\
\hline Mobile only with helper & 3 & $12 \cdot 0$ & 5 & $13 \cdot 2$ & 6 & $24 \cdot 0$ & \\
\hline Mobile alone & 22 & 88.0 & 33 & $86 \cdot 8$ & 17 & $68 \cdot 0$ & \\
\hline
\end{tabular}

T1, before fracture; T3, at hospital discharge; T4, 6 months after T3.

${ }^{*}$ Results for the $\leq 25 \%$ and $>25-50 \% \mathrm{DI}$ groups were significantly different from those of the $>50 \% \mathrm{DI}$ group $\left(P<0.05 ; \chi^{2}\right.$ or Fisher's exact test after correction for multiple tests in pairwise comparisons: Benjamini-Hochberg procedure).

$\dagger P$ for differences between all three groups by $\chi^{2}$ or Fisher's exact test. 
Table 4. Clinical course of geriatric hip fracture patients according to postoperative dietary intake (DI) (Number of patients and percentages; median values with their 25th-75th percentiles)

\begin{tabular}{|c|c|c|c|c|c|c|c|}
\hline & \multicolumn{7}{|c|}{ DI level } \\
\hline & \multicolumn{2}{|c|}{$>50 \%$} & \multicolumn{2}{|c|}{$>25-50 \%$} & \multicolumn{2}{|c|}{$\leq 25 \%$} & \multirow[b]{2}{*}{$P \ddagger$} \\
\hline & $n$ & $\%$ & $n$ & $\%$ & $n$ & $\%$ & \\
\hline Subjects $(n)$ & \multicolumn{2}{|c|}{25} & \multicolumn{2}{|c|}{38} & \multicolumn{2}{|c|}{25} & \\
\hline Length of hospital stay (d) & \multirow{2}{*}{\multicolumn{2}{|c|}{11}} & & & & & 0.403 \\
\hline Median & & & \multicolumn{2}{|c|}{13} & \multicolumn{2}{|c|}{11} & \\
\hline 25th-75th percentile & \multicolumn{2}{|c|}{$9-14$} & \multicolumn{2}{|c|}{$9-17$} & \multicolumn{2}{|c|}{$8-15$} & \\
\hline Patients with complications until T3 & 21 & $84 \cdot 0$ & 36 & $94 \cdot 7$ & 25 & $100 \cdot 0$ & 0.075 \\
\hline Complications (no. per patient) & & & & & & & $0.012^{*}$ \\
\hline Median & \multicolumn{2}{|c|}{2} & \multicolumn{2}{|c|}{3} & \multicolumn{2}{|c|}{3} & \\
\hline 25th-75th percentile & \multicolumn{2}{|c|}{$1-3$} & \multicolumn{2}{|c|}{$2-4$} & \multicolumn{2}{|c|}{$3-4$} & \\
\hline Electrolyte imbalance & 18 & $72 \cdot 0$ & 25 & $65 \cdot 8$ & 16 & $64 \cdot 0$ & 0.879 \\
\hline Infections & 8 & 32.0 & 18 & 47.4 & 17 & 68.0 & 0.036 \\
\hline Urinary tract & 6 & 24.0 & 16 & $42 \cdot 1$ & 16 & $64 \cdot 0$ & $0.017^{*}$ \\
\hline Gastrointestinal & 3 & $12 \cdot 0$ & 2 & $5 \cdot 3$ & 0 & & 0.174 \\
\hline Other & 2 & 8.0 & 3 & 7.9 & 3 & $12 \cdot 0$ & 0.897 \\
\hline Delirium & 9 & $36 \cdot 0$ & 20 & $52 \cdot 6$ & 10 & $40 \cdot 0$ & 0.413 \\
\hline Severe anaemia & 3 & $12 \cdot 0$ & 8 & $21 \cdot 1$ & 8 & $32 \cdot 0$ & 0.243 \\
\hline Acute renal insufficiency & 2 & 8.0 & 9 & 23.7 & 6 & 24.0 & 0.273 \\
\hline New pressure ulcers & 3 & $12 \cdot 0$ & 8 & $21 \cdot 1$ & 5 & $20 \cdot 0$ & 0.689 \\
\hline Falls & 2 & 8.0 & 5 & 13.2 & 4 & $16 \cdot 0$ & 0.783 \\
\hline Gastrointestinal (not infections) & 0 & & 7 & $18 \cdot 4$ & 4 & $16 \cdot 0$ & 0.069 \\
\hline Wound or implant related & 2 & 8.0 & 4 & $10 \cdot 5$ & 3 & $12 \cdot 0$ & 0.999 \\
\hline Cardiovascular & 2 & 8.0 & 1 & $2 \cdot 6$ & 5 & $20 \cdot 0$ & 0.061 \\
\hline Dehydration & 1 & 4.0 & 0 & & 6 & $24 \cdot 0$ & $0.002 \dagger$ \\
\hline Additional operation & 1 & 4.0 & 3 & 7.9 & 1 & 4.0 & 0.999 \\
\hline Cerebrovascular event & 0 & & 2 & $5 \cdot 3$ & 0 & & 0.504 \\
\hline Other & 1 & 4.0 & 3 & 7.9 & 0 & & 0.567 \\
\hline Patients with rehospitalisation until T4 & 10 & $40 \cdot 0$ & 17 & 44.7 & 13 & 52.0 & 0.683 \\
\hline
\end{tabular}

T3, at hospital discharge; T4, 6 months after T3.

${ }^{*}$ Results for the $\leq 25 \%$ DI group were significantly different from those of the $>50 \%$ DI group $(P<0.05$; MannWhitney $U, \chi^{2}$ or Fisher's exact test after correction for multiple tests in pairwise comparisons: Benjamini-Hochberg procedure).

† Results for the $\leq 25 \% \mathrm{DI}$ group were significantly different from those of the $>25-50 \% \mathrm{DI}$ group $(P<0.05$; MannWhitney $U, \chi^{2}$ or Fisher's exact test after correction for multiple tests in pairwise comparisons: Benjamini-Hochberg procedure).

$\ddagger P$ for differences between all groups by Kruskal-Wallis, $\chi^{2}$ or Fisher's exact test.

underestimation of absolute DI. Snacks provided by relatives and oral nutritional supplements were recorded when noticed but we decided not to include these data into DI estimation, as systematic monitoring was not guaranteed. However, according to the records taken, snacks or supplements were generally consumed very rarely, leading to the conclusion that underestimation should be only marginal. Moreover, this information bias can be assumed to be largely the same for all three groups and thus should not lead to systematic error, although this cannot be known for sure. In another study including patients from all ages with all kinds of diseases, it was estimated that in hospitals the intake from snacks and supplements provides about $25-30 \% / \mathrm{d}$ of extra energy and protein ${ }^{(22)}$. In view of the fact that the three main meals provided daily by hospital catering contained on average $8400 \mathrm{~kJ}(2000 \mathrm{kcal})$ and $80 \mathrm{~g}$ protein $/ \mathrm{d}$, mean daily energy and protein intake per patient from main meals in the present study can very roughly be estimated as $<2100 \mathrm{~kJ}$ and $<20 \mathrm{~g}$ protein/d when DI $\leq 25 \%$, and as $<4200 \mathrm{~kJ}$ and $<40 \mathrm{~g}$ protein/d when DI $>25-50 \%$. Even if an additional daily intake from snacks and supplements of about $30 \%$ is assumed, this would only result in approximately $<2700 \mathrm{~kJ}$ and $<26 \mathrm{~g}$ protein/d, and about $<5400 \mathrm{~kJ}$ and $<52 \mathrm{~g}$ protein/d, respectively, which would still be far below most patients' requirements ${ }^{(48)}$.

Such a very low postoperative DI in geriatric HF patients has previously been found in other studies, where an average intake between $2780 \mathrm{~kJ} / \mathrm{d}$ over the first three postoperative days $^{(27)}$ and $3965 \mathrm{~kJ} / \mathrm{d}$ over the first postoperative week $^{(26)}$ was reported (calculated from estimated portion ingested of all food items and snacks served). Anbar et al. ${ }^{(24)}$ observed a mean energy intake from food of $3250 \mathrm{~kJ} / \mathrm{d}$ plus $400 \mathrm{~kJ} / \mathrm{d}$ from supplements and a mean protein intake of $37 \mathrm{~g} / \mathrm{d}$ (assessed with food records) over the whole hospital stay (13 (SD 6) d). Considering that other studies ${ }^{(19,33)}$ reported mean energy intakes from 4220 to $4340 \mathrm{~kJ} / \mathrm{d}$ at admission to rehabilitation 2-4 weeks after surgery, it can be assumed that also in many of our patients DI remained very low after the fourth postoperative day. Further, taking into account that most patients of the present study already went through a prolonged period of mandatory fasting before surgery (median 33 (25th-75th percentile 20-47) h, which is comparable to reports from other HF studies ${ }^{(24,26)}$ ), without much doubt in most of our participants DI was far from sufficient to cover the basic nutritional needs for at least 1 week and probably even longer. 
There are other studies that report a slightly higher mean DI of $4600-5400 \mathrm{~kJ} / \mathrm{d}$ and up to $1 \mathrm{~g}$ protein $/ \mathrm{kg}$ body weight $/ \mathrm{d}$ over the first seven to fourteen postoperative days ${ }^{(25,29,30)}$. However, in these studies, none of the participants was malnourished before HF or was cognitively impaired, whereas the results of the present study show that significantly more patients with low postoperative DI were already malnourished before HF, had lost weight and/or had reduced DI, and all of them were at least mildly cognitively impaired after surgery. Excluding such patients thus might lead to records of higher DI, as seen in the aforementioned reports.

The high prevalence of malnutrition and cognitive impairment in patients with low DI (or vice versa) reflects a general state of frailty and reveals the highly worrying fact that precisely the most vulnerable patients seem to be predisposed to low postoperative DI (which also might be a sign for a generally low DI). Comparable findings have been reported by other groups ${ }^{(8,19,20,25,31,32)}$, indicating that nutritional problems that occurred before HF (not surprisingly) continued or exacerbated in hospital, and that these problems were not properly addressed by hospital care. The cumulating energy and nutrient deficit caused by inadequate DI (before and) throughout hospital stay can very likely be assumed to contribute substantially to these patients' reduced capacity for rehabilitation ${ }^{(11)}$

In accord with this hypothesis, in the present study, longterm impairments in ADL and mobility were seen significantly more often and more pronounced in patients with lowest postoperative DI. Such a relation has been reported as early as 1983 by Bastow et $a l{ }^{(32)}$, who found that patients with lower DI (mean $4200 \mathrm{~kJ} / \mathrm{d}$ from fifth postoperative day to discharge after about $40 \mathrm{~d}$, calculated from estimated portion ingested of all food items and snacks served) needed more time to restore independent mobility than patients with a higher DI (that was either achieved voluntarily or by supplementary nightly enteral tube feeding). In 2000, Paillaud et $a l .{ }^{(18)}$ observed the same relation in patients of a rehabilitation clinic, where those patients with lowest voluntary DI about 3 weeks after surgery needed considerably more time to self-sufficient mobility than those with highest DI. In contrast, other studies found no significant differences in functional development according to $\mathrm{DI}^{(26,28,30,33)}$. However, all previous studies only had short follow-up periods and were all primarily designed to evaluate the effect of increasing DI through supplementation, and therefore did not differentiate their control groups in patients with higher or lower voluntary DI. To our knowledge, this is the first time that differences in voluntary postoperative DI were analysed for their influence on functional development after HF over four points in time until 6 months after hospitalisation and including patients from all functional and cognitive levels. In most previous studies, the exclusion of relevant patient subgroups like functionally or cognitively impaired patients ${ }^{(18,32,33)}$ or malnourished persons ${ }^{(30,33)}$ most likely led to the exclusion of those with lowest DI and probably worst functional recovery.

The patients with lowest DI also suffered from significantly more postoperative in-hospital complications, thus adding to the growing body of evidence that insufficient DI is associated with a worse postoperative clinical course, especially with the development of postoperative infections ${ }^{(24,27-29)}$. However, all postoperative nutritional intervention studies to prevent such complications up to now yielded only conflicting results ${ }^{(11,49)}$, and thus it has to be taken into account that association does not necessarily implicate causality. Foss et $a l .{ }^{(35)}$ correctly stated that it should also be considered that suffering from complications may lead to insufficient DI. Moreover, other factors such as the deleterious impact of $\mathrm{HF}$ and surgery on the general health of these patients might influence the prevalence of both, and the same effect has of course to be taken into consideration for postoperative functional development too. Certainly, more well-designed high-quality trials addressing and elucidating this relationship are needed, preferably including clinical and biochemical data from the short-term recovery period, in order to shed more light on this aspect.

Length of hospital stay and rehospitalisation did not differ significantly between DI groups. However, in the German disease-related group reimbursement system currently established in the health care system, both parameters are hugely influenced by a variety of factors (e.g. minimal and maximal reimbursed length of stay per diagnosis) and do not always primarily reflect the individual patients' condition. Due to differences in health care system structures, such results are also hardly comparable between countries, sometimes not even between hospitals ${ }^{(50)}$.

Of course, it is not only nutritional deficits that cause unfavourable functional outcomes, but in the present study, the consideration of potential confounders through ANCOVA did not change the result that postoperative DI has a significant influence on functional development after HF. This underlines that nutrition is one important contributing factor in the rehabilitation process, and that nutritional deficits have to be addressed as part of a complex care concept considering all relevant factors that affect rehabilitation success ${ }^{(6,7)}$.

\section{Limitations}

One major limitation of the present study is that the statistical power for detecting differences between DI groups is limited by the rather small overall sample size. Another problem is that we could not systematically assess and analyse the patients' individual reasons for low DI, which would be key information for the development of suitable, patient-tailored nutritional interventions to positively influence DI. Study staff impression is that among the reasons heard most often from patients, relatives and ward staff, 'refuses to eat' (especially in those cognitively impaired), 'no appetite', 'don't like the food', 'cannot chew the food', 'nausea', 'not feeling well' and 'too tired' were prominent, but also 'didn't get the help needed'. These and many more reasons for low DI in hospitalised geriatric HF patients have also been detected and discussed rather comprehensively by other groups $^{(11,20,22,51,52)}$. Sadly, as seen again in the present study, even today, nutritional deficits frequently remain undetected in clinical routine ${ }^{(11,15)}$. As there is hardly any situation more convenient to initiate nutritional therapies than the 
hospital setting, suitable interventions ideally should start there and continue in rehabilitation. To cope with the challenges presented to health care systems by demographic change, it is necessary to be more cost-effective by 'making the most' of the hospitalisation period with its high inevitable fixed costs by providing optimal treatment that minimises follow-up costs ${ }^{(53)}$.

\section{Conclusions}

In the present study, significantly more patients with lower postoperative DI turned out to have already been in a state of frailty before $\mathrm{HF}$, as they were malnourished and cognitively impaired, indicating that the most vulnerable patients seem to be highly predisposed to low postoperative DI.

Obviously, this subgroup of patients would need to be in the focus of care and get specific nutritional support to achieve adequate DI. Ideally, any therapy should not only help to maintain their functional capacity by covering present nutritional needs, but should also compensate for previously accumulated nutritional deficits. Therefore, future research will need to focus on suitable, patient-tailored nutritional intervention strategies that help to achieve adequate DI in the vulnerable group of malnourished older HF patients to support their functional and clinical recovery, thereby elucidating which kind of therapeutic concepts are most beneficial for these patients regarding functional and clinical course after HF.

\section{Acknowledgements}

The authors are grateful to all study participants and their proxies as well as the staff at the orthogeriatric and other Departments of Trauma and Orthopaedic Surgery and the hospital kitchen at Klinikum Nürnberg for their cooperation and support. We also wish to thank Christin Ender (Nutritional Scientist) and Katja Purucker (MD) for their very valuable help in conducting the present study.

This research received no specific grant from any funding agency, commercial or not-for-profit sectors. S. G. and D. V. were supported by the Theo and Friedl Schöller-Foundation, Nürnberg, Germany, and S. G. was scholarship holder of a Bavarian Equal Opportunities Sponsorship (Förderung von Frauen in Forschung und Lehre - Promoting Equal Opportunities for Women in Research and Teaching) of the Friedrich-Alexander-Universität Erlangen-Nürnberg, Germany. These funding sources had no role in the design or analysis of the study or the writing of this article.

The authors declare that there are no known conflicts of interest associated with this publication. The primary author is a current $\mathrm{PhD}$ candidate. The present work was performed in partial fulfilment of the requirements for obtaining the degree 'Doktor der Humanbiologie (Dr rer. biol. hum.)' at the Friedrich-Alexander-Universität Erlangen-Nürnberg, Germany.

The authors' contributions are as follows: S. G. was primarily responsible for patient recruitment, data acquisition, analysis and interpretation, and for drafting of the manuscript. E. S. contributed to study conception and data interpretation.
K. S. contributed to study conception, data acquisition and interpretation. O. G. contributed to data analysis and manuscript revision. T. B., R. B., H.-J. B. and C. C. S. assisted with study conception and patient recruitment. D. V. was mainly responsible for study design and conception and contributed to data interpretation and analysis as well as to manuscript drafting and revision. The final version of this manuscript has been approved by all named authors, and there are no other persons who fulfilled the criteria for authorship but are not listed.

\section{References}

1. Konnopka A, Jerusel N \& König H (2009) The health and economic consequences of osteopenia- and osteoporosisattributable hip fractures in Germany: estimation for 2002 and projection until 2050. Osteoporos Int 20, 1117-1129.

2. Roth T, Kammerlander C, Gosch M, et al. (2010) Outcome in geriatric fracture patients and how it can be improved. Osteoporos Int 21, 615-619.

3. Gill TM, Murphy TE, Gahbauer EA, et al. (2013) The course of disability before and after a serious fall injury. JAMA Intern Med 173, 1780-1786.

4. Vochteloo AJH, Moerman S, Tuinebreijer WE, et al. (2013) More than half of hip fracture patients do not regain mobility in the first postoperative year. Geriatr Gerontol Int $\mathbf{1 3}$, 334-341.

5. Baker PN, Salar O, Ollivere BJ, et al. (2014) Evolution of the hip fracture population: time to consider the future? A retrospective observational analysis. BMJ Open 4, e004405.

6. Inzitari M, Doets E, Bartali B, et al. (2011) Nutrition in the age-related disablement process. J Nutr Health Aging 15, 599-604.

7. Fiatarone Singh MA (2014) Exercise, nutrition and managing hip fracture in older persons. Curr Opin Clin Nutr Metab Care 17, 12-24.

8. Murphy MC, Brooks CN, New SA, et al. (2000) The use of the Mini-Nutritional Assessment (MNA) tool in elderly orthopaedic patients. Eur J Clin Nutr 54, 555-562.

9. Li H, Cheng H, Liang J, et al. (2013) Functional recovery of older people with hip fracture: does malnutrition make a difference? J Adv Nurs 69, 1691-1703.

10. Koren-Hakim T, Weiss A, Hershkovitz A, et al. (2012) The relationship between nutritional status of hip fracture operated elderly patients and their functioning, comorbidity and outcome. Clin Nutr 31, 917-921.

11. Corish CA \& Kennedy NP (2000) Protein-energy undernutrition in hospital in-patients. BrJ Nutr 83, 575-591.

12. World Health Organization (1995) Physical Status: The Use and Interpretation of Anthropometry. WHO Technical Report Series no. 854. Geneva: WHO pp. 1-452.

13. Sorensen J, Kondrup J, Prokopowicz J, et al. (2008) EuroOOPS: an international, multicentre study to implement nutritional risk screening and evaluate clinical outcome. Clin Nutr 27, 340-349.

14. Goisser S, Schrader E, Singler K, et al. (2015) Malnutrition according to Mini Nutritional Assessment (MNA) is associated with severe functional impairment in geriatric patients before and up to 6 months after hip fracture. J Am Med Dir Assoc (Epublication ahead of print version 9 April 2015).

15. Volkert D, Saeglitz C, Gueldenzoph H, et al. (2010) Undiagnosed malnutrition and nutrition-related problems in geriatric patients. J Nutr Health Aging 14, 387-392. 
16. McWhirter JP \& Pennington CR (1994) Incidence and recognition of malnutrition in hospital. BMJ 308, 945-948.

17. Cansado P, Ravasco P \& Camilo M (2009) A longitudinal study of hospital undernutrition in the elderly: comparison of four validated methods. J Nutr Health Aging 13, 159-164.

18. Paillaud E, Bories PN, Le Parco JC, et al. (2000) Nutritional status and energy expenditure in elderly patients with recent hip fracture during a 2-month follow-up. BrJ Nutr 83, 97-103.

19. Nematy M, Hickson M, Brynes AE, et al. (2006) Vulnerable patients with a fractured neck of femur: nutritional status and support in hospital. J Hum Nutr Diet 19, 209-218.

20. Bell J, Bauer J, Capra S, et al. (2013) Barriers to nutritional intake in patients with acute hip fracture: time to treat malnutrition as a disease and food as a medicine? Can J Physiol Pharmacol 91, 489-495.

21. Thibault R, Chikhi M, Clerc A, et al. (2011) Assessment of food intake in hospitalised patients: a 10-year comparative study of a prospective hospital survey. Clin Nutr 30, 289-296.

22. Dupertuis Y, Kossovsky MP, Kyle UG, et al. (2003) Food intake in 1707 hospitalised patients: a prospective comprehensive hospital survey. Clin Nutr 22, 115-123.

23. Hiesmayr M, Schindler K, Pernicka E, et al. (2009) Decreased food intake is a risk factor for mortality in hospitalised patients: the NutritionDay survey 2006. Clin Nutr 28, 484-491.

24. Anbar R, Beloosesky Y, Cohen J, et al. (2014) Tight calorie control in geriatric patients following hip fracture decreases complications: a randomized, controlled study. Clin Nutr 33, $23-28$.

25. Hoekstra JC, Goosen JH, de Wolf SG, et al. (2011) Effectiveness of multidisciplinary nutritional care on nutritional intake, nutritional status and quality of life in patients with hip fractures: a controlled prospective cohort study. Clin Nutr 30, 455-461.

26. Sullivan DH, Nelson CL, Klimberg VS, et al. (2004) Nightly enteral nutrition support of elderly hip fracture patients: a pilot study. J Am Coll Nutr 23, 683-691.

27. Eneroth M, Olsson U \& Thorngren K (2006) Nutritional supplementation decreases hip fracture-related complications. Clin Orthop Relat Res 451, 212-217.

28. Gunnarsson A, Lönn K \& Gunningberg L (2009) Does nutritional intervention for patients with hip fractures reduce postoperative complications and improve rehabilitation? J Clin Nurs 18, 1325-1333.

29. Delmi M, Rapin CH, Bengoa JM, et al. (1990) Dietary supplementation in elderly patients with fractured neck of the femur. Lancet 335, 1013-1016.

30. Botella-Carretero JI, Iglesias B, Balsa JA, et al. (2010) Perioperative oral nutritional supplements in normally or mildly undernourished geriatric patients submitted to surgery for hip fracture: a randomized clinical trial. Clin Nutr 29, 574-579.

31. Lumbers M, New SA, Gibson S, et al. (2001) Nutritional status in elderly female hip fracture patients: comparison with an age-matched home living group attending day centres. $\mathrm{Br} \mathrm{J}$ Nutr 85, 733-740.

32. Bastow MD, Rawlings J \& Allison SP (1983) Benefits of supplementary tube feeding after fractured neck of femur: a randomised controlled trial. Br Med J (Clin Res Ed) 287 , 1589-1592.

33. Myint MW, Wu J, Wong E, et al. (2012) Clinical benefits of oral nutritional supplementation for elderly hip fracture patients: a single blind randomised controlled trial. Age Ageing 42, 39-45.

34. Seitz DP, Adunuri N, Gill SS, et al. (2011) Prevalence of dementia and cognitive impairment among older adults with hip fractures. J Am Med Dir Assoc 12, 556-564.
35. Foss NB, Jensen PS \& Kehlet H (2007) Risk factors for insufficient perioperative oral nutrition after hip fracture surgery within a multi-modal rehabilitation programme. Age Ageing 36, 538-543.

36. DIMDI - Deutsches Institut für Medizinische Dokumentation und Information (German Institute of Medical Documentation and Information) (2013) International Classification of Diseases, 10th Revision, German Modification (ICD-10-GM), Version 2013. Cologne: DIMDI. http://www.dimdi.de/static/ en/klassi/icd-10-gm/index.htm (accessed September 2013).

37. Charlson ME, Pompei P, Ales KL, et al. (1987) A new method of classifying prognostic comorbidity in longitudinal studies: development and validation. J Chronic Dis 40, 373-383.

38. Guigoz Y, Vellas B \& Garry PJ (1994) Mini Nutritional Assessment: a practical assessment tool for grading nutritional state of elderly patients. Facts Res Gerontol 4, Suppl. 2, 15-59.

39. Folstein MF, Folstein SE \& McHugh PR (1975) "Mini-mental state". A practical method for grading the cognitive state of patients for the clinician. J Psychiatr Res 12, 189-198.

40. Loveman E, Green C, Kirby J, et al. (2006) The clinical and cost-effectiveness of donepezil, rivastigmine, galantamine and memantine for Alzheimer's disease. Health Technol Assess 10, 1-160.

41. Yesavage JA, Brink TL, Rose TL, et al. (1982) Development and validation of a geriatric depression screening scale: a preliminary report. J Psychiatr Res 17, 37-49.

42. Bjornsdottir R, Oskarsdottir ES, Thordardottir FR, et al. (2013) Validation of a plate diagram sheet for estimation of energy and protein intake in hospitalized patients. Clin Nutr 32, 746-751.

43. Goisser S, Schrader E, Ender C, et al. (2013) PP036-Sun is an estimated plate diagram suitable to correctly assess postoperative dietary intake of geriatric patients with hip fracture? Clin Nutr 32, Suppl. 1, S18.

44. Mahoney F \& Barthel D (1965) Functional evaluation: the Barthel index. Md State Med J 14, 61-65.

45. Benjamini Y \& Hochberg Y (1995) Controlling the false discovery rate: a practical and powerful approach to multiple testing. J R Stat Soc B Meth 57, 289-300.

46. Gumieiro DN, Rafacho BPM, Gonçalves AF, et al. (2013) Mini Nutritional Assessment predicts gait status and mortality 6 months after hip fracture. Br J Nutr 109, 1657-1661.

47. Castellanos VH \& Andrews YN (2002) Inherent flaws in a method of estimating meal intake commonly used in longterm-care facilities. J Am Diet Assoc 102, 826-830.

48. Miller MD, Daniels LA, Bannerman E, et al. (2005) Resting energy expenditure measured longitudinally following hip fracture compared to predictive equations: is an injury adjustment required? Br J Nutr 94, 976-982.

49. Avenell A \& Handoll HHG (2010) Nutritional supplementation for hip fracture aftercare in older people. The Cochrane Database of Systematic Reviews 2010, issue 1, CD001880.

50. Liem IS, Kammerlander C, Suhm N, et al. (2013) Identifying a standard set of outcome parameters for the evaluation of orthogeriatric co-management for hip fractures. Injury $\mathbf{4 4}$, 1403-1412.

51. Nieuwenhuizen WF, Weenen H, Rigby P, et al. (2010) Older adults and patients in need of nutritional support: review of current treatment options and factors influencing nutritional intake. Clin Nutr 29, 160-169.

52. Patel MD \& Martin FC (2008) Why don't elderly hospital inpatients eat adequately? J Nutr Health Aging 12, 227-231.

53. Wyers CE, Reijven PL, Evers SM, et al. (2013) Cost-effectiveness of nutritional intervention in elderly subjects after hip fracture. A randomized controlled trial. Osteoporos Int 24, $151-162$. 